\title{
Study on molecular imaging and radionuclide therapy of human nasopharyngeal carcinoma cells transfected with baculovirus-mediated sodium/iodine symporter gene
}

\author{
JIANZHANG WANG ${ }^{1}$, SHUAI LIU ${ }^{2}$, JUN WANG ${ }^{1}$, YIFAN ZHANG ${ }^{2}$, \\ BIAO $\mathrm{LI}^{2}$, CHANGPING CAI ${ }^{1}$ and SHILI WANG ${ }^{1}$ \\ Departments of ${ }^{1}$ Otolaryngology and ${ }^{2}$ Nuclear Medicine, Ruijin Hospital, Shanghai Jiaotong \\ University School of Medicine, Shanghai 200025, P.R. China
}

Received February 27, 2013; Accepted April 19, 2013

DOI: $10.3892 /$ ijo.2013.1936

\begin{abstract}
The non-invasive imaging and radiotherapy by sodium/iodine symporter (NIS) gene transfer have been widely used for many experiments and some clinical studies. Baculovirus is an efficient tool for gene delivery into mammalian cells in vitro and in vivo. However, the applications of NIS and/or baculovirus in nasopharyngeal carcinoma (NPC) cells have not been reported yet. In this study, two recombinant baculoviruses expressing, respectively, NIS and green fluorescent protein (GFP), both under the control of the cytomegalovirus promoter (Bac-NIS and Bac-GFP) were successfully constructed. The infection efficiency and GFP fluorescence intensity of the human NPC cell line CNE-2Z infected by Bac-GFP at different setting of multiplicity of infection (MOI) were determined by flow cytometry. NIS protein expression was detected by indirect immunofluorescence. The ${ }^{125} \mathrm{I}$ uptake and efflux of infected CNE-2Z cells by Bac-NIS were measured by a $\gamma$-counter. The cytotoxicity of baculovirus and sodium butyrate and inhibition of iodine uptake by $\mathrm{NaClO}_{4}$ were examined. The radioactivity and GFP fluorescence intensity in co-infected CNE-2Z cells by Bac-NIS and Bac-GFP were measured. Cell colony formation tests were conducted to evaluate the killing effect of Bac-NIS-mediated ${ }^{131}$ I. Based on the results, the transduction efficiency of Bac-GFP at the MOI of 200 or 400 reached 91.16 and $94.79 \%$, respectively. NIS protein was expressed accurately on transfected CNE-2Z cell membranes and performed its normal function in iodine transport. Baculovirus had hardly any cytotoxic effects on infected cells, while relatively high concentration of sodium butyrate generated cytotoxicity. The
\end{abstract}

Correspondence to: Professor Shili Wang, Department of Otolaryngology, Ruijin Hospital, Shanghai Jiaotong University School of Medicine, 197 Rui Jin 2nd Road, Shanghai 200025, P.R. China E-mail: shiliwangruijin@hotmail.com

Key words: human nasopharyngeal carcinoma, baculovirus, sodium/iodine symporter, molecular imaging, radionuclide therapy, gene therapy correlation coefficient between the GFP fluorescence intensity and radioactivity in co-infected CNE-2Z cells was 0.917 . Treatment coupled Bac-NIS with ${ }^{131}$ I killed the infected tumour cells dramatically in vitro. These results suggest that baculovirus is an effective vector of the gene delivery into CNE-2Z cells and NIS-mediated iodine transport is a potential approach for molecular imaging and radionuclide therapy of NPC.

\section{Introduction}

The sodium/iodine symporter (NIS) is an intrinsic plasma membrane glycoprotein and responsible for thyroidal, gastric, salivary, intestinal and mammary iodide transport. The rat NIS gene was first cloned in 1996 (1), human NIS (hNIS) gene later in the same year (2) and mouse NIS gene in 2001 (3). Cloning and characterization of the NIS gene make it possible to deliver this gene into non-thyroid cells. NIS can be used with both diagnostic $\left({ }^{125} \mathrm{I},{ }^{124} \mathrm{I}, 99 \mathrm{mTc}\right)$ and therapeutic $\left({ }^{131} \mathrm{I},{ }^{211} \mathrm{At},{ }^{186} \mathrm{Re}\right.$, ${ }^{188} \mathrm{Re}$ ) radioisotopes, and the potential value of NIS usage has become more important for researchers. There has been great progress in discovering the biological function of NIS in thyroid and non-thyroid tissues (4), which results in increasing use of NIS as a means of achieving imaging or therapeutic goals not only in thyroid cancer but also in non-thyroidal tumour tissues.

NIS gene has been applied to a series of viral and non-viral vectors in which it can be used for live animal imaging. Transient or stable NIS expression in non-thyroidal tissues and tumour xenografts promoting iodide uptake has led to the use of NIS as an imaging reporter in many preclinical experiments and some clinical studies. The three main research areas of NIS reporter imaging are viral-mediated gene therapy, oncolytic viral therapy and cell trafficking (e.g., stem cell delivery) (5). NIS is one of the most promising radionuclide reporters for preclinical and translational research.

The characteristics of the NIS gene indicate that it can serve as a good therapeutic gene for cancer therapy. NIS has been used as a therapeutic gene for therapy of thyroid cancer and hyperthyroidism for a long time (6). Importantly, NIS has a high bystander effect, whereby non-transduced tumour cells can be caught in the electron cross-fire emanating from the transduced cells and still eradicated by the emission of an electron 
( $\beta$ particle) (7). Moreover, because NIS is a normal human gene and protein, its expression in target cells is unlikely to be toxic or limit its efficacy in patients. In view of these advantages, the field of NIS gene therapy has made considerable strides. Researchers have demonstrated the feasibility of NIS mediated gene therapy in vitro and/or in vivo for various tumours such as thyroid, breast, liver, prostate, pancreas, colorectal, colon, ovarian and uterine cervical carcinomas, and multiple myeloma (8).

Nasopharyngeal carcinoma (NPC) has a unique geographical and ethnic predisposition with high incidences in Southern China, Southeast Asia, the Middle East/North Africa and the Arctic. P53 protein plays a vital role in regulating the cell cycle, differentiation and apoptosis, and may also have an important therapeutic implication (9). Because NPC is highly radiosensitive, radiotherapy is the primary treatment. In recent years, its treatment has achieved great progress including gene therapy, such as a recombinant adenovirus expressing p53 in 2003 (10). However, novel efficient diagnosis and therapeutic approaches still need to be established in the management of patients with recurrent, residual or metastatic disease (11). It is significant to monitor the in vivo distribution, replication and elimination of replicating vectors as well as therapeutic gene expression in the NPC gene therapy. As mentioned above, NIS gene transfer has the ability of in vivo imaging and therapy for the radiosensitive tumours. Therefore, it is meaningful to explore the radiosensitivity of the NPC cells mediated by NIS.

Effective cancer gene therapy depends on efficient viral infection as well as persistent gene expression in the target tissue, which needs an ideal gene transfer vector. Due to the biosafety, huge cloning capacity, low cytotoxicity and non-replication nature in the target cells and the ease of manipulation and production, baculovirus has emerged as a novel vector for in vitro and in vivo gene delivery among a variety of viral and non-viral vectors (12). However, there have been no report of NPC cells transducted by baculovirus. Although serum inactivation of baculovirus is a significant barrier to the development of this vector for therapeutic gene delivery, NPC has the advantage of the unique location where baculovirus can be injected directly, accurately and repeatedly into the target site of the tumour from the air, which can avoid baculovirus inactivation in the presence of serum complement to some extent.

To investigate the applicability of NIS and baculovirus in NPC therapy and prepare for the next in vivo gene therapy, we produced the recombinant baculoviruses Bac-GFP and Bac-NIS, and conducted a series of related experiments on an human NPC cell line CNE-2Z with the above viruses in vitro.

\section{Materials and methods}

Plasmids and cells. Plasmid pFastBac-CMV-GFP was kindly provided by the Institute of Molecular Biology, The University of Hong Kong; pFastBac 1 vector was obtained from Invitrogen; pcDNA-hNIS was a gift of Dr Sissy Jhiang at the Ohio State University; Sf9 cells from Invitrogen were cultured in Sf900 III medium with $10 \%$ fetal bovine serum (FBS); human NPC line CNE-2Z, purchased from Jiangyin K.K. Company, were cultured in RPMI-1640 medium supplemented with $10 \%$ FBS and $1 \%$ penicillin/streptomycin in a humidified environment with $5 \% \mathrm{CO}_{2}$ at $37^{\circ} \mathrm{C}$.
Construction of plasmid pFastBac-CMV-hNIS. Polyhedron $(\mathrm{PH})$ promoter gene in pFastBac 1 vector was replaced with cytomegalovirus (CMV) promoter gene, and then plasmid pFastBac-CMV was generated. Human NIS (hNIS) gene was obtained from pcDNA-NIS with the restriction enzymes SalI and Xbal. Next, hNIS gene fragment and pFastBac-CMV vector were digested simultaneously with $S a l \mathrm{I}$ and $\mathrm{Xbal}$, and connected by T4 DNA ligase. Plasmid pFastBac-CMV-hNIS was constructed successfully after verification by agarose gel electrophoresis and sequencing.

Production of recombinant baculoviruses. Plasmids pFastBac-CMV-hNIS and pFastBac-CMV-GFP were transformed into DH10Bac E. coli to generate recombinant bacmids, which were later transfected into Sf9 cells to generate recombinant baculoviruses. After amplification, we generated successfully Bac-NIS (Bac-CMV-hNIS) and Bac-GFP (Bac-CMV-GFP). The titer of a baculoviral stock was determined through performing a viral plaque assay. Guidelines and instructions were referred to Bac-to-Bac Baculovirus Expression System manual.

Infection efficiency and fluorescence intensity of Bac-GFP and indirect immunofluorescence of Bac-NIS. CNE-2Z cells were seed in 24-well plates at a density of $5 \times 10^{4}$ cells/well and cultured until they were $80 \%$ confluent at the time of infection. The initial media were replaced with PBS including Bac-GFP at MOIs of 0, 50, 100, 200 and 400 with or without $5 \mathrm{mmol} / \mathrm{l}$ sodium butyrate just before infection, which was changed to RPMI with $10 \%$ FBS $4 \mathrm{~h}$ later. To test the effect of sodium butyrate, CNE-2Z cells were infected by Bac-GFP at MOI of 100 and different concentrations of sodium butyrate were added. The infection efficiency and the mean fluorescence intensity of Bac-GFP group were determined by flow cytometry. Bac-NIS group was infected by Bac-NIS at MOI of 200 and stained with indirect immunofluorescence (anti-NIS goat polyclonal antibody from Santa Cruz Company, 1:200 and DyLight594 goat anti-rabbit IgG from MultiSciences Company, 1:200) after $24 \mathrm{~h}$ incubation. Then, cells in Bac-GFP or Bac-NIS group were observed by an inversion fluorescence microscope. The excitation wavelength of blue laser was $488 \mathrm{~nm}$ and the detection wavelength was $520 \mathrm{~nm}$. All the following infections of CNE-2Z cells by Bac-NIS or Bac-GFP were treated following the above process and performed in triplicate in this experiment.

The cytotoxicity of recombinant baculovirus and sodium butyrate. CNE-2Z cells were seeded in 96-well plates at a density of $5 \times 10^{3}$ cells/well and cultured until they were $80-90 \%$ confluent before infection. For Bac-GFP group, Bac-GFP at MOIs of $0,100,200,400,600$ or 800 was added. For sodium butyrate group, Bac-GFP at a MOI of 400 and sodium butyrate at the concentrations of $0,5,10,15,20$ or $25 \mathrm{mmol} / \mathrm{l}$ were added. In addition, control group was free of Bac-GFP and sodium butyrate, and blank group was treated with only RPMI without cells, Bac-GFP and sodium butyrate. Twenty-four hours later, initial media were replaced with fresh RPMI, $10 \mu \mathrm{l}$ of CCK-8 was added into each well and incubated for another $4 \mathrm{~h}$. The absorption at $450 \mathrm{~nm}$ (A450) was measured with a microplate reader. Cell viability was calculated with the equa- 

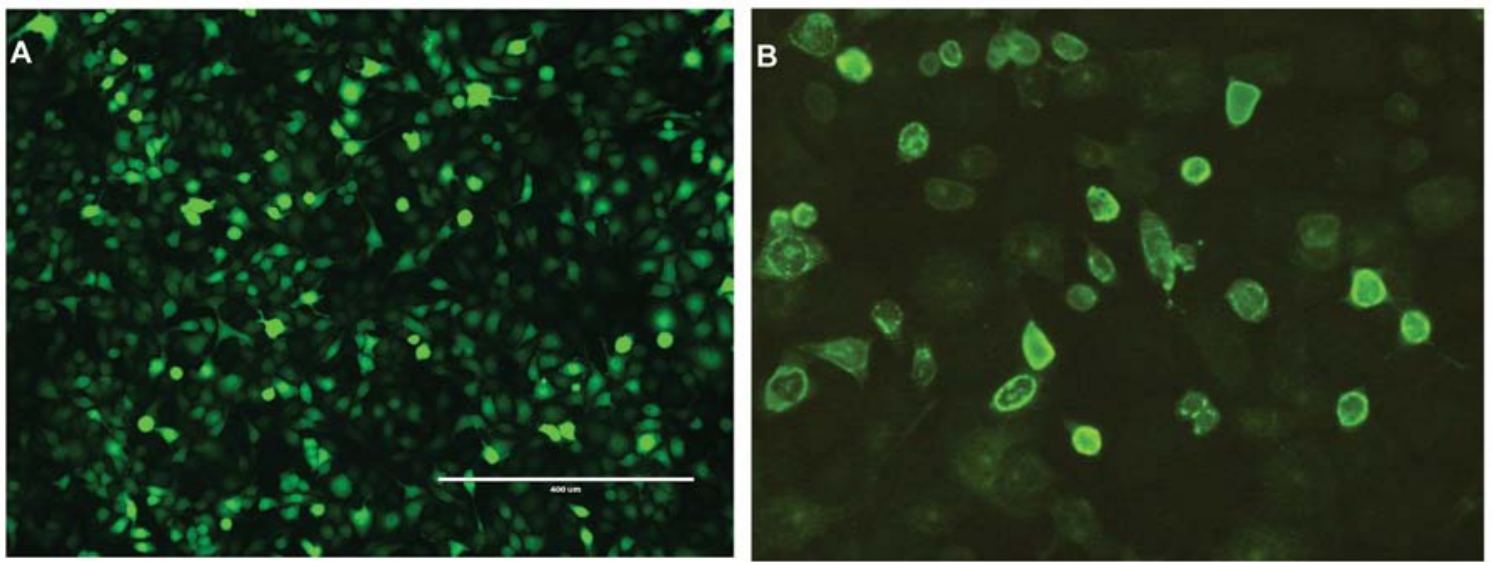

Figure 1. The expression of GFP and NIS in infected CNE-2Z cells. (A) Abundant GFP expression in Bac-GFP infected CNE-2Z cells. (B) The detection of NIS protein by immunofluorescence on Bac-NIS infected CNE-2Z cell membranes.

tion: cell viability $(\%)=($ test well A450 - blank well A450)/ (control well A450 - blank well A450) x 100\%.

${ }^{125}$ I uptake assay. The assay was performed as described (13). CNE-2Z cells were infected by Bac-NIS at MOIs of 0, 25, 50, 100, 200 or 400, and washed twice by Hank's balanced salt solution (HBSS) $24 \mathrm{~h}$ later. To each well was added $0.1 \mu \mathrm{Ci}$ $\mathrm{Na}^{125} \mathrm{I}$ and $1 \mu \mathrm{M}$ NaI in a total volume of $0.5 \mathrm{ml} \mathrm{HBSS}$ and incubated at $37^{\circ} \mathrm{C}$ for $30 \mathrm{~min}$. Next, cells were washed 3 times with ice-cold HBSS, and then $0.5 \mathrm{ml} 100 \%$ ice-cold dehydrated alcohol was added. Twenty minutes later, the radioactivity (counts per minute, cpm) in each well was measured by a $\gamma$-counter.

Dynamic ${ }^{125}$ I uptake. After 24 h of the Bac-NIS or Bac-GFP infection both at MOI of 200, ${ }^{125}$ I uptake of CNE-2Z cells was measured as depicted above at $0,5,10,15,30,45,60,75,90$, 105 and 120 min after $\mathrm{Na}^{125} \mathrm{I}$ and $\mathrm{NaI}$ incubation, respectively.

${ }^{125}$ I efflux test. ${ }^{125} \mathrm{I}$ efflux was measured as previously reported (13). Following $45 \mathrm{~min}$ of $\mathrm{Na}^{125} \mathrm{I}$ and $\mathrm{NaI}$ incubation of the CNE-2Z cells infected with Bac-NIS at MOI of 200, the cell-culture medium was replaced with fresh HBSS every 5 min and further incubated for in $30 \mathrm{~min}$. The cells were treated with $0.5 \mathrm{ml}$ dehydrated alcohol at $30 \mathrm{~min}$ to measure the residual radioactivity in the cells. At the same time, the buffers replaced at different times were measured.

NIS gene expression time of duration. To test NIS gene expression time, CNE-2Z cells were infected with Bac-NIS or Bac-GFP at MOI of 400 , the ${ }^{125} \mathrm{I}$ uptake was measured as depicted above at days 1, 2, 4, 8, 12, 16 and 20. During that period of time, cell culture medium was refreshed every 3 days.

Inhibition of iodine uptake by $\mathrm{NaClO}_{4}$. Twenty-four hours following infection of CNE-2Z cells with Bac-NIS at MOI of 200, $0.1 \mu \mathrm{Ci} \mathrm{Na}{ }^{125} \mathrm{I}, 1 \mu \mathrm{M} \mathrm{NaI}$ and $\mathrm{NaClO}_{4}$ at concentrations of $0,10,20,50,100$ or $200 \mu \mathrm{mol} / 1$ in a total volume of $0.5 \mathrm{ml}$ HBSS were added to each well, 45 min later, CNE-2Z cells were washed, lysed and counted as described above.
Correlation between radioactivity and fluorescence intensity in co-infected CNE-2Z cells with Bac-NIS and Bac-GFP. This experiment was performed to explore the correlation between ${ }^{125} \mathrm{I}$ uptake and fluorescence intensity in co-infected CNE-2Z cells. CNE-2Z cells were co-infected with Bac-NIS and Bac-GFP at total viral MOIs of 0, 50, 100, 200 or 400, and each MOI of each virus for every well was equal at MOIs of $0,25,50,100$ or 200 , respectively. Twenty-four hours after infection, 3 wells of each MOI were tested by flow cytometry to determine the mean fluorescence intensity. The cells of 3 wells of each MOI were tested by a $\gamma$-counter to measure iodine uptake.

${ }^{131}$ I-mediated killing of CNE-2Z cells in cell colony formation test. To estimate the killing effect of CNE-2Z cells by ${ }^{131} \mathrm{I}$, a cell colony formation test including six groups was performed. CNE-2Z cells in all groups were seed in 24-well plates at a density of $1 \times 10^{5}$ cells/well and cultured for $24 \mathrm{~h}$. Next, group 1 (Bac-NIS $+{ }^{131} \mathrm{I}$ ) and group 4 (Bac-NIS) were infected with Bac-NIS at MOI of 400; group 2 (Bac-GFP+ $\left.{ }^{131} \mathrm{I}\right)$ and group 5 (Bac-GFP) were infected with Bac-GFP at MOI of 400; group 3 (HBSS $\left.+{ }^{131} \mathrm{I}\right)$ and group 6 (HBSS) were treated with HBSS in the corresponding way. Twenty-four hours later, all the cells were washed twice by HBSS. To each well in groups 1 , 2 and 3 were added $50 \mu \mathrm{Ci} \mathrm{Na}{ }^{131} \mathrm{I}$ in a total volume of $0.5 \mathrm{ml}$ HBSS and incubated for $6 \mathrm{~h}$, while groups 4,5 and 6 were free of $\mathrm{Na}^{131} \mathrm{I}$. Then, all the cells were washed twice with HBSS, trypsinized, counted, plated in a 6-well plate (300 cells/well), and incubated. During colony growth, the culture medium was replaced every 3 days. At the 7 th day, cells were washed three times with HBSS and stained with $0.1 \%$ crystal violet solution. The colony was counted only if it contained more than 30 cells in a well. Colony formation rate $(\%)=$ (number of colonies/number of plated cells) x $100 \%$.

Statistical analysis. Each treatment was carried out in triplicates, unless noted otherwise. Statistical analysis was performed using SPSS 21.0 software. All data are presented as the mean \pm SD. Differences were considered significant at $\mathrm{P}<0.05$. 
A

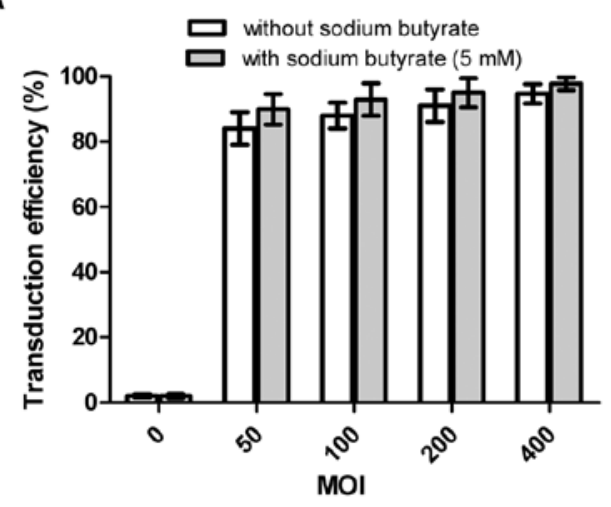

C

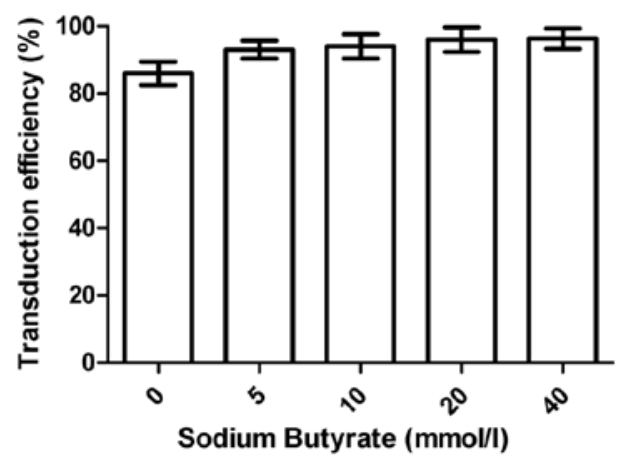

B

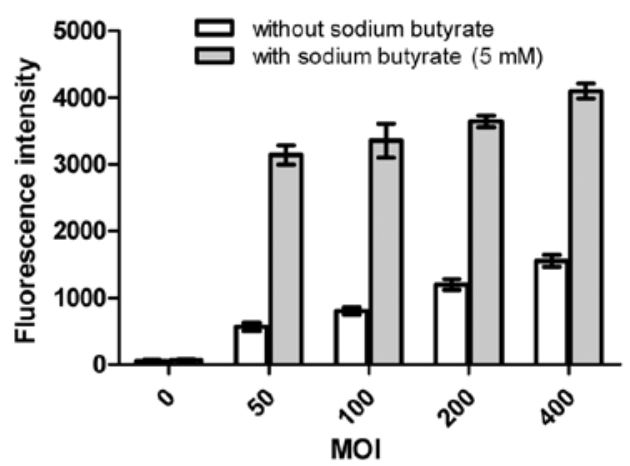

D

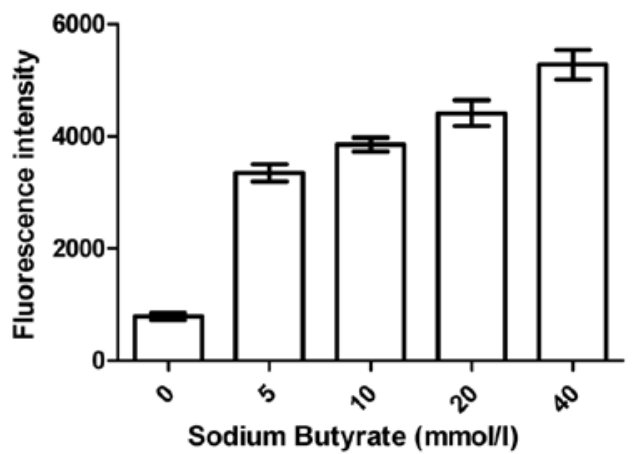

Figure 2. Transduction efficiency and fluorescence intensity of Bac-GFP in CNE-2Z cells at different MOIs with or without sodium butyrate. (A) Transduction efficiency and (B) fluorescence intensity of Bac-GFP at MOIs of 0, 50, 100, 200 and 400 with or without 5 mmol/1 sodium butyrate. (C) Transduction efficiency and (D) fluorescence intensity of Bac-GFP at MOI of 100 with various concentrations of sodium butyrate at $0,5,10,20$ and 40 mmol/1.

\section{Results}

Immunofluorescence of Bac-NIS. The stocks of Bac-NIS and Bac-GFP were $2 \times 10^{9}$ and $5 \times 10^{9} \mathrm{pfu} / \mathrm{ml}$, respectively. As can be seen from the Fig. 1B, the NIS protein was clearly and precisely expressed on CNE-2Z cell membranes, which indicated that Bac-NIS expressed NIS protein in infected CNE-2Z cells efficiently. Correspondingly, Fig. 1A depicts abundant GFP expression in CNE-2Z cells infected with Bac-GFP.

Transduction efficiency and fluorescence intensity of Bac-GFP with or without sodium butyrate. As can be seen from the Fig. 2, both the transduction efficiency and fluorescence intensity of Bac-GFP in the CNE-2Z cells became stronger with increasing MOIs, whilst the former saw a gentle upward trend and the later surged when $5 \mathrm{mmol} / \mathrm{l}$ or different concentrations of sodium butyrate were added. Additionally, there were no statistically significant differences for the transduction efficiency between groups with or without sodium butyrate whether at different MOIs of Bac-GFP or various concentrations of sodium butyrate, whereas, the corresponding results of fluorescence intensity were the opposite, and the differences were statistically significant. It is noteworthy that the transduction efficiency of Bac-GFP at the MOI of 400 without or with $5 \mathrm{mmol} / \mathrm{l}$ sodium butyrate reached 94.79 and $97.86 \%$, respectively.
The cytotoxicity of baculovirus and sodium butyrate. Fig. 3A demonstrates that the cell survival rate in Bac-GFP group had no significant decrease with increasing MOIs. Even at MOI of 800 , there was no obvious cell death. Therefore, baculovirus had hardly any cytotoxic effects on the CNE-2Z cells. As shown in Fig. 3B, the cell survival rate in sodium butyrate group decreased obviously with increasing concentrations of sodium butyrate with Bac-GFP at MOI of 400. The cell viability changed significantly when the concentration of sodium butyrate was higher than $10 \mathrm{mmol} / \mathrm{l}$. The above suggested that the baculovirus itself did not decrease the CNE-2Z cell viability significantly, while relatively high concentration of sodium butyrate generated cytotoxicity on the cells.

${ }^{125}$ I uptake at different MOIs. Fig. 4 shows that the cpm in the infected CNE-2Z cells changed with the increasing MOIs of Bac-NIS. It shot up over the first MOI of 100, peaked at MOI of 200 and then showed a slight downward trend. Therefore, CNE-2Z cells were infected with Bac-NIS at MOI of 200 in the following dynamic ${ }^{125}$ I uptake test.

Dynamic ${ }^{125}$ I uptake at MOI of 200. Fig. 5 indicates CNE-2Z cells absorbed ${ }^{125} \mathrm{I}$ in relation to incubation time. The first $45 \mathrm{~min}$ witnessed a dramatic rise, with cpm peaking at 11,200 and cpm in the following $75 \mathrm{~min}$ saw a fall from the peak at $45 \mathrm{~min}$ to only 4,200 at $120 \mathrm{~min}$. The ${ }^{125}$ I uptake of Bac-NIS group was 70 -fold higher than that of Bac-GFP group. 

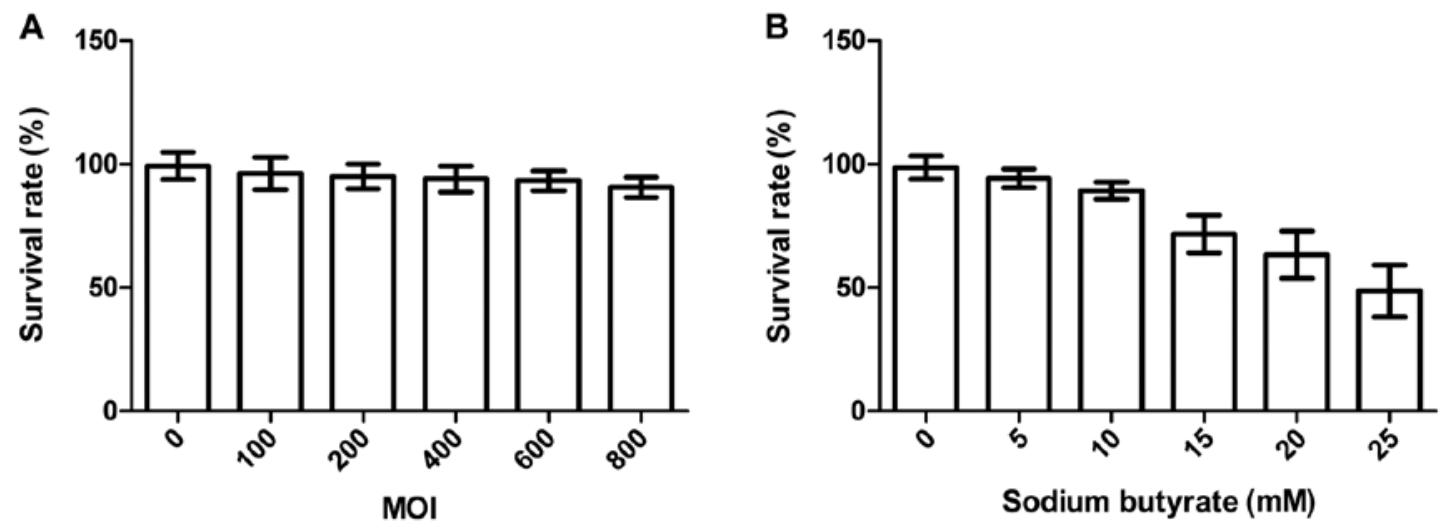

Figure 3. Cytotoxic effects of baculovirus and sodium butyrate on CNE-2Z cells. (A) The survival rate of CNE-2Z cells after infection of Bac-GFP at MOIs of 0, $100,200,400,600$ and 800 . (B) The survival rate of CNE-2Z cells after infection of Bac-GFP at MOI of 400 with various concentrations of sodium butyrate at $0,5,10,20$ and $25 \mathrm{mmol} / \mathrm{l}$.

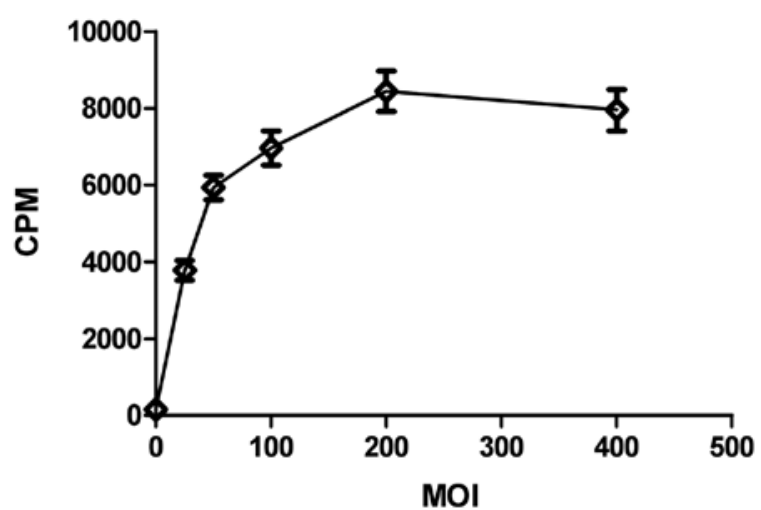

Figure 4. ${ }^{125}$ I uptake mediated by Bac-NIS in CNE-2Z cells at different MOIs. Iodine uptake (cpm) was determined in CNE-2Z cells infected with Bac-NIS at MOIs of 0, 25, 50, 100, 200 and 400, 30 min after exposure to $\mathrm{Na}^{125} \mathrm{I}$ and $\mathrm{NaI}$.

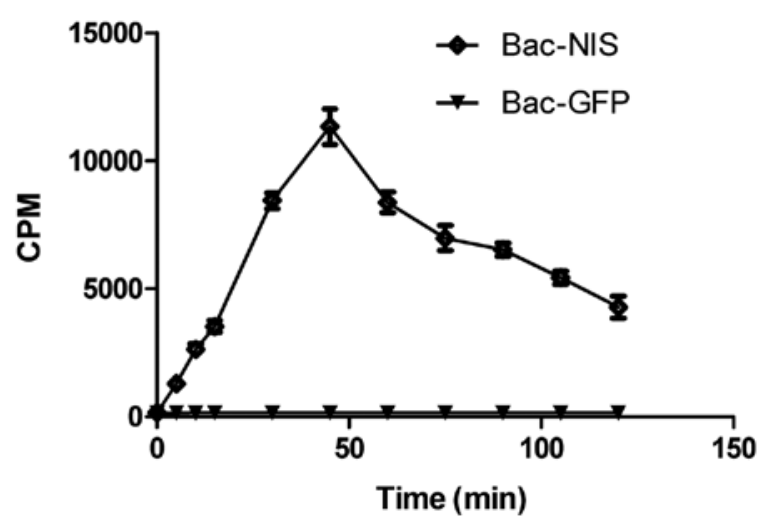

Figure 5. Dynamic ${ }^{125}$ I uptake mediated by Bac-NIS in CNE-2Z cells. Iodine uptake in infected CNE-2Z cells by Bac-NIS at MOI of 200 was determined at different times of $\mathrm{Na}^{125} \mathrm{I}$ and $\mathrm{NaI}$ incubation.

${ }^{125}$ I efflux test. Fig. 6 shows the continuous outflow of ${ }^{125} \mathrm{I}$ into the medium as time went on. Nearly half of the total cellular iodine was released in the initial $10 \mathrm{~min}$ and only

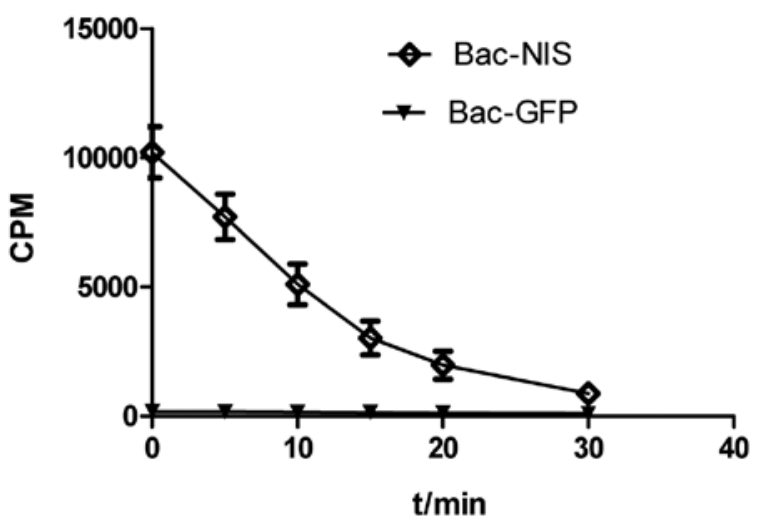

Figure 6. ${ }^{125}$ I efflux mediated by Bac-NIS in CNE-2Z cells. Iodine efflux in CNE-2Z cells mediated by Bac-NIS at MOI of 200 was determined 45 min after incubation with $\mathrm{Na}^{125} \mathrm{I}$ and $\mathrm{NaI}$.

less than $10 \%$ remained in infected cells at the time of $30 \mathrm{~min}$.

NIS gene expression time of duration. After CNE-2Z cells were infected with Bac-NIS at MOI of 400 , the ${ }^{125}$ I uptake was measured at different days. The results in Fig. 7 describe that the cpm leveled off relatively over the first 4 days, descended gently between the 4 th and 8 th day, and reached the bottom at day 20 , which indicated that the expression of NIS was stable within 8 days after infection.

Inhibition of $\mathrm{NaClO}_{4}$. Fig. 8 illustrates that the impact of $\mathrm{NaClO}_{4}$ on ${ }^{125} \mathrm{I}$ uptake of CNE-2Z cells was obvious, with 87.5, 90.1, 91.5, 92.6 and $94.0 \%$ of ${ }^{125} \mathrm{I}$ uptake inhibited along with increasing concentrations of $\mathrm{NaClO}_{4}$, which demonstrated that the function of the NIS protein in the CNE-2Z cells was also inhibited by the presence of $\mathrm{NaClO}_{4}$ as in thyroid tissue.

Correlation between ${ }^{125}$ I uptake and GFP fluorescence intensity in co-infected CNE- $2 Z$ cells. The correlation between cpm and GFP fluorescence intensity is depicted in Fig. 9, which displays a definite trend, and the correlation coefficiency between them 


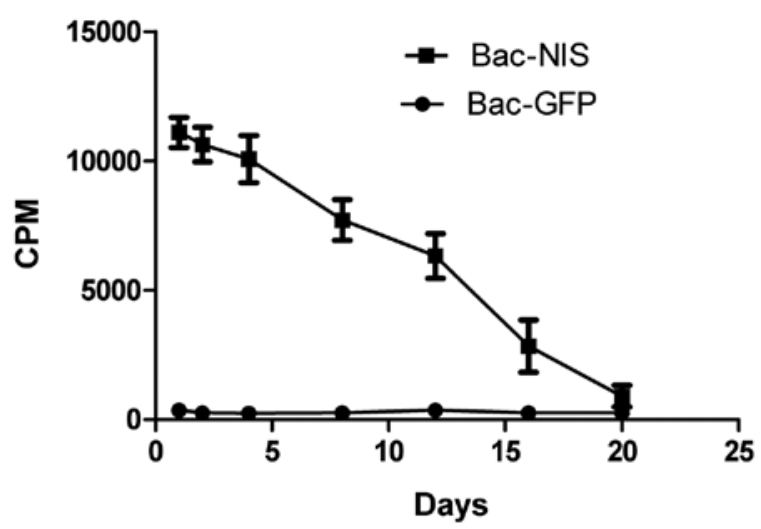

Figure 7. NIS gene expression duration in infected CNE-2Z cells. Iodine uptake in infected CNE-2Z cells by Bac-NIS or Bac-GFP at MOI of 400 was measured at days $1,2,4,8,12,16$ and 20.

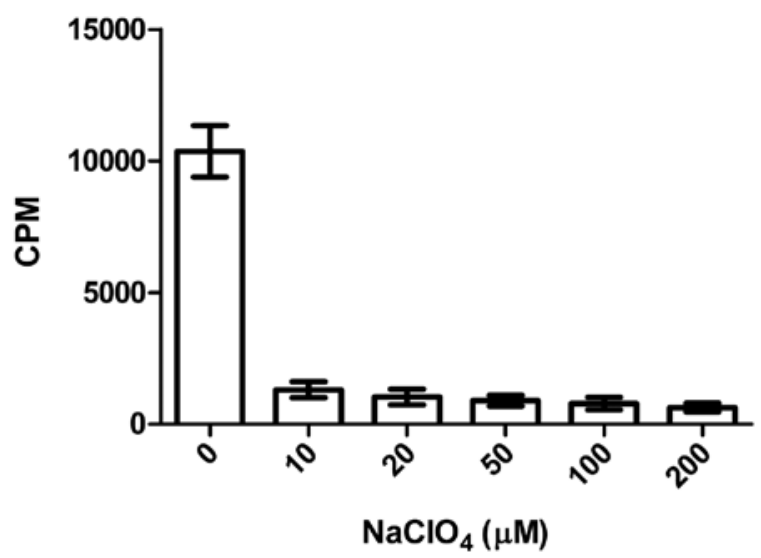

Figure 8. Inhibition effect of $\mathrm{NaClO}_{4}$ in iodine uptake in infected CNE-2Z cells. Iodine uptake was measured $45 \mathrm{~min}$ after $\mathrm{NaClO}_{4}$ at concentrations of 0 , $10,20,50,100$ or $200 \mu \mathrm{mol} / 1$ was added with $\mathrm{Na}^{125} \mathrm{I}$ and $\mathrm{NaI}$.

was 0.917 , a high positive correlation. The above data show that the GFP expression level is highly correlated to that of NIS, so the level of radioactivity mirrored the GFP expression level.

The cell colony formation test. A cell colony formation test can evaluate the killing effect of ${ }^{131} \mathrm{I}$ in infected tumour cells and the results of the colony-forming assay show the survival rate. The results in Fig. 10 indicate that the cell survival rate of the group Bac-NIS with ${ }^{131}$ I was $20.7 \%$, which was substantially lower than that of the other five groups $(\mathrm{P}<0.01)$. The cell viability in group $2(66.3 \%)$ was similar to that of the group $3(67.6 \%)$. Cell survival rates of the three groups without ${ }^{131}$ I were virtually equal, which also indicated baculovirus had scarcely any impact on cell survival. Therefore, the treatment of Bac-NIS with ${ }^{131}$ I can kill infected CNE-2Z cells efficiently in vitro.

\section{Discussion}

Baculovirus can transduce a broad range of mammalian and avian cells (14), and mediate efficient gene expression in a wide range of vertebrate cells (15). In this study, we constructed

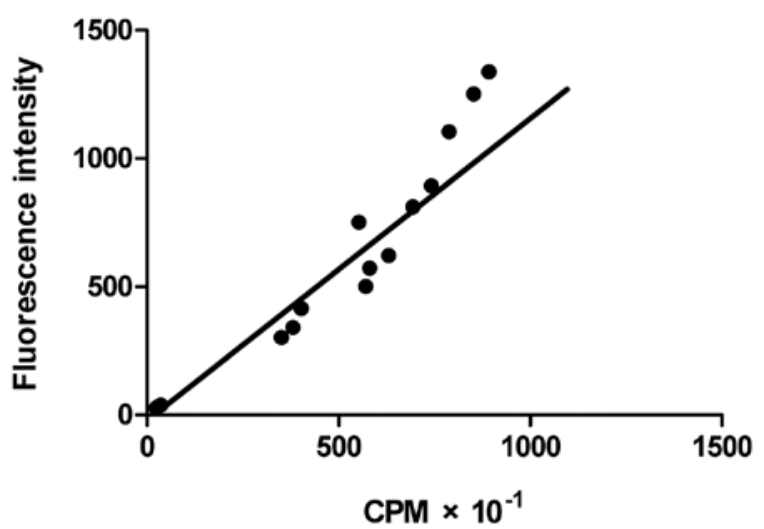

Figure 9. The correlation between ${ }^{125} \mathrm{I}$ uptake and GFP fluorescence intensity in co-infected CNE-2Z cells. CNE-2Z cells were co-infected with Bac-NIS and Bac-GFP at total viral MOIs of 0, 50, 100, 200 or 400 (each MOI of each virus was equal at MOIs of $0,25,50,100$ or 200, respectively). Fluorescence intensity and iodine uptake were measured $24 \mathrm{~h}$ after co-infection, respectively. Coefficiency $\mathrm{R}^{2}=0.917$.

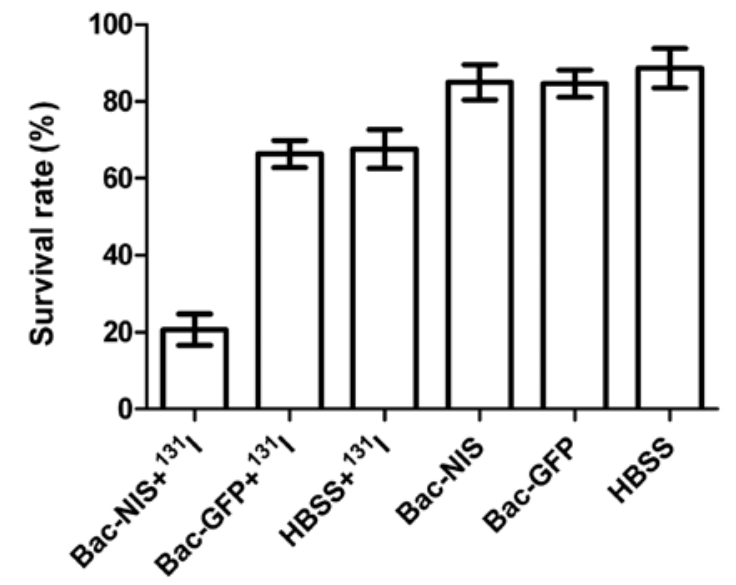

Figure 10. Survival rates of tumour cells with different treatments. CNE-2Z cells were divided into six groups and the killing effect of ${ }^{131} \mathrm{I}$ was tested by a cell colony-forming assay.

Bac-GFP and Bac-NIS with high titre successfully and the transduction rate of baculovirus on CNE-2Z cells reached $91.16 \%$ at MOI of 200. The radioiodine uptake of the infected CNE-2Z cells by Bac-NIS at the MOI of 200 reached the peak at $45 \mathrm{~min}$ and was significantly reduced with escalating doses of $\mathrm{NaClO}_{4}$, which indicated that the NIS protein expressed in NPC cells could perform its normal function in iodine uptake just as in thyroid cells. High infection efficiency, low cytotoxicity, durable NIS expression, a physiological protein without immunogenicity theoretically, and easiness to obtain radioactive iodine $\left({ }^{131} \mathrm{I},{ }^{125} \mathrm{I},{ }^{124} \mathrm{I}\right.$, etc.) and technetium are favorable factors for NIS gene imaging and therapy of NPC.

For quantitative use in visualization and analyzation by PET and SPECT, the NIS gene has gained a broad application in the non-invasive imaging approach monitoring cell migration, localization, proliferation and other biological processes or observing the real-time level and duration of target gene expression (16). It is extremely advantageous if gene therapy vectors can achieve the reliable non-invasive monitoring of vector 
biodistribution and population together with therapeutic gene expression, which is critical for the evaluation of the success or failure of gene therapy approaches. However, most therapeutic genes cannot be quantified directly by using molecular imaging just as NIS gene, so the NIS reporter gene and a therapeutic gene can be combined to perform indirect imaging (17). In this study, we adopted co-infection approach in which CNE-2Z cells were co-infected with Bac-GFP and Bac-NIS at the same MOIs, and the result showed correlation coefficiency of 0.917 between the fluorescence intensity and radioactivity. A similar way has been reported (18). Consequently, there is a correlation between GFP and NIS expression in CNE-2Z cells and we can use the changes in the level of radioactivity to assess changes in GFP expression level. Furthermore, the way of co-infection is an effective approach to fulfil multi-gene co-expression in the target cells when GFP gene is replaced by a desired gene.

The effective ${ }^{131} \mathrm{I}$ therapy of most differentiated thyroid cancer has been conformed more than 60 years and the field of radiotherapy by NIS gene transfer has made huge leaps in recent years (19). The cell colony formation test demonstrated that NIS-mediated CNE-2Z cells could be effectively and specifically killed by ${ }^{131} \mathrm{I}$, which laid a good foundation for the application of NIS in NPC. Furthermore, ${ }^{188} \mathrm{Re}$ (Rhenium) radiating $\beta$-rays (20) and ${ }^{211}$ At (Astatine) radiating $\alpha$-rays (21) mediated by NIS have even better radiotherapeutic effects than ${ }^{131}$ I for their higher radiation energy, which are stronger weapons for NIS-mediated cancer gene therapy suggesting that NIS gene is a promising candidate for NPC radiotherapy.

Since the discovery that baculovirus could enter mammalian cells and mediate transgene expression under the control of mammalian promoters (22), recombinant baculovirus vectors have become an efficient tool for gene transfer into mammalian cells (12). Of particular concern is serum inactivation of gene transduction in vivo mediated by baculovirus (23). However, the locus of NPC is another favorable factor for gene therapy. Bac-NIS can be injected directly, repeatedly and accurately into the local region of the tumour when needed, which can avoid circulation in the blood and have a good targeting property. Therefore, this can escape serum inactivation to a certain extent. In addition, sodium butyrate as a histone deacetylase inhibitor can obviously enhance infection efficiency of baculovirus, while it has some cytotoxicity (24), we also confirmed this conclusion above. The results above indicated that sodium butyrate improved the efficiency of baculovirus infection in CNE-2Z cells but that this effect was not quite statistically significant. Having high infection rate without sodium butyrate and the convenience of treatment, recombinant baculovirus is an appropriate gene delivery vector for use in NPC.

Radiotherapy is the standard treatment for NPC, and it can produce a variety of complications for the location of the tumour at the base of skull and in close proximity to radiation dose-limiting organs, giving brachytherapy an advantage in this aspect (15). NIS can mediate active ${ }^{131} \mathrm{I}$ transport and ${ }^{131} \mathrm{I}$ has been used successfully to treat thyroid disorders for a long time. Given this and the unique feature of ${ }^{131} \mathrm{I}$, the ability of Bac-NIS to accumulate ${ }^{131}$ I may be applied in NPC brachytherapy with its unique advantages: the amount and time of ${ }^{131} \mathrm{I}$ injection can be adjusted; accurate application is still feasible even in the irregular contour of the nasopharynx; NIS can serve as an imaging reporter at the same time. All of the above data show Bac-NIS has a wide application prospect in NPC brachytherapy.

Although radiation and chemotherapy in the treatment of NPC are effective, local failure or regional failure presenting as persistent or recurrent tumour is still inevitable. Early detection is of great concern for any form of salvage therapy. As is well known that it is difficult to confirm residual or recurrent tumour in the cervical lymph nodes after radiotherapy (25). In addition, durable responses are rarely encountered in metastatic disease and the recurrent tumours are more resistant to chemotherapy after initial responses in cytotoxic chemotherapy. However, the combination Bac-NIS and mesenchymal stem cells (MSC) has the potential to solve these problems because the adoptively transferred MSCs can migrate and engraft into the stroma of established tumour lesions (26). MSCs as a cellular delivery vehicle with specific tropisms has been exploited in some tumours (27). Importantly, the infection efficiency of recombinant baculovirus is quite high in MSCs (28). In addition, not only would MSCs protect baculovirus from serum complement attack, but also they would allow the amplification of the initial viral load with the possibility of increasing viral spread through cell-to-cell contact specifically at tumour sites (27). Therefore, MSCs as a vehicle for targeted delivery of NIS to tumours may be applied in NPC, and a relative study reported the image-guided and tumour stroma-targeted ${ }^{131} \mathrm{I}$ therapy of hepatocellular cancer after systemic MSCs-mediated NIS gene delivery (29). Therefore, this approach is not limited to radiologically and reliably detectable tumours, but can also be used to target minimal residual, recurrent and micrometastatic tumours, which demonstrates a better prospect of application in NPC for the above problems. In this context, the approach of MSCs-mediated therapeutic gene with easy mode of administration (intravenous injection) may in the future provide the means for therapy of NPC and our study establishes a solid foundation for this.

In this study, CNE-2Z cells were first infected with Bac-GFP and Bac-NIS. We found that baculovirus was able to penetrate and deliver target genes into CNE-2Z cells and the transduction rate reached as high as $94.79 \%$ at MOI of 400 , which not only expanded the transduction range of baculovirus, but also proved the feasibility of gene delivery mediated by baculovirus in NPC. The high related coefficient between ${ }^{125} \mathrm{I}$ uptake and GFP fluorescence intensity in co-infected CEZ-2Z cells and ${ }^{131}$ I-mediated killing of tumour cells demonstrated that Bac-NIS could be applied in molecular imaging and radioactive therapy in NPC. Therefore, we believe the combination Bac-NIS has bright prospects in diagnosis and treatment of NPC.

\section{Acknowledgements}

This study was supported with research funds (114119a6400) from the Science and Technology Commission of Shanghai Municipality, China.

\section{References}

1. Dai G, Levy O and Carrasco N: Cloning and characterization of the thyroid iodide transporter. Nature 379: 458-460, 1996.

2. Smanik P, Liu Q, Furminger T, et al: Cloning of the human sodium iodide symporter. Biochem Biophys Res Commun 226: 339-345, 1996. 
3. Pinke L, Dean D, Bergert E, Spitzweg C, Dutton C and Morris J: Cloning of the mouse sodium iodide symporter. Thyroid 11: 935-939, 2001

4. Dohán O, De la Vieja A, Paroder V, et al: The sodium/iodide symporter (NIS): characterization, regulation, and medical significance. Endocr Rev 24: 48-77, 2003.

5. Penheiter AR, Russell SJ and Carlson SK: The sodium iodide symporter (NIS) as an imaging reporter for gene, viral, and cell-based therapies. Curr Gene Ther 12: 33-47, 2012.

6. Carvalho DP and Ferreira AC: The importance of sodium/ iodide symporter (NIS) for thyroid cancer management. Arq Bras Endocrinol Metabol 51: 672-682, 2007.

7. Ferlini C, Amelio RD and Scambia G: Apoptosis induced by ionizing radiation. The biological basis of radiosensitivity. Subcell Biochem 36: 171-186, 2002.

8. Kogai T and Brent GA: The sodium iodide symporter (NIS): Regulation and approaches to targeting for cancer therapeutics. Pharmacol Ther 135: 355-370, 2012

9. Sun Y, Yi H, Yang Y, et al: Functional characterization of p53 in nasopharyngeal carcinoma by stable shRNA expression. Int J Oncol 34: 1017-1027, 2009.

10. Peng Z: Current status of gendicine in China: recombinant human Ad-p53 agent for treatment of cancers. Hum Gene Ther 16: 1016-1027, 2005

11. Li X, Liu X, Li C, et al: Recombinant adeno-associated virus mediated RNA interference inhibits metastasis of nasopharyngeal cancer cells in vivo and in vitro by suppression of Epstein-Barr virus encoded LMP-1. Int J Oncol 29: 595-603, 2006.

12. Airenne KJ, Mahonen AJ, Laitinen OH and Yla-Herttuala S: Baculovirus-mediated gene transfer: an emerging universal concept. In: Gene and Cell Therapy: Therapeutic Mechanisms and Strategies. Smith Templeton N (ed). Chapter 11. 3rd edition. CRC Press, Boca Raton, FL, pp263-281, 2008.

13. Weiss SJ, Philp NJ and Grollman EF: Iodide transport in a continuous line of cultured cells from rat thyroid. Endocrinology 114: 1090-1098, 1984.

14. Chen CY, Lin CY, Chen GY and Hu YC: Baculovirus as a gene delivery vector: recent understandings of molecular alterations in transduced cells and latest applications. Biotechnol Adv 29: 618-631, 2011.

15. Wang C, Busse J and Gitterman M: A simple afterloading applicator for intracavitary irradiation of carcinoma of the nasopharynx. Radiology 115: 737-738, 1975.

16. Dingli D, Russell SJ and Morris JC III: In vivo imaging and tumor therapy with the sodium iodide symporter. J Cell Biochem 90: 1079-1086, 2003.
17. Blasberg RG: Molecular imaging and cancer. Mol Cancer Ther 2: 335-343, 2003.

18. Yaghoubi S, Wu L, Liang Q, et al: Direct correlation between positron emission tomographic images of two reporter genes delivered by two distinct adenoviral vectors. Gene Ther 8: 1072-1080, 2001

19. Hingorani M, Spitzweg C, Vassaux G, et al: The biology of the sodium iodide symporter and its potential for targeted gene delivery. Curr Cancer Drug Targets 10: 242-267, 2010

20. Shen D, Marsee D, Schaap J, et al: Effects of dose, intervention time, and radionuclide on sodium iodide symporter (NIS)-targeted radionuclide therapy. Gene Ther 11: 161-169, 2004.

21. Willhauck MJ, Samani B-RS, Wolf I, et al: The potential of 211 Astatine for NIS-mediated radionuclide therapy in prostate cancer. Eur J Nucl Med Mol Imaging 35: 1272-1281, 2008.

22. Hofmann C, Sandig V, Jennings G, Rudolph M, Schlag P and Strauss M: Efficient gene transfer into human hepatocytes by baculovirus vectors. Proc Natl Acad Sci 92: 10099-10103, 1995.

23. Kaikkonen MU, Ylä-Herttuala S and Airenne KJ: How to avoid complement attack in baculovirus-mediated gene delivery. J Invertebr Pathol 107: S71-S79, 2011.

24. Hu YC, Tsai CT, Chang YJ and Huang JH: Enhancement and prolongation of baculovirus mediated expression in mammalian cells: focuses on strategic infection and feeding. Biotechnol Prog 19: 373-379, 2008.

25. Wei WI, Ho CM, Wong MP, Fung Ng W, Lau SK and Lam KH: Pathological basis of surgery in the management of postradiotherapy cervical metastasis in nasopharyngeal carcinoma. Arch Otolaryngol Head Neck Surg 118: 923-930, 1992.

26. Studeny M, Marini FC, Champlin RE, Zompetta C, Fidler IJ and Andreeff M: Bone marrow-derived mesenchymal stem cells as vehicles for interferon- $\beta$ delivery into tumors. Cancer Res 62: 3603-3608, 2002.

27. Hall B, Andreeff $M$ and Marini F: The participation of mesenchymal stem cells in tumor stroma formation and their application as targeted-gene delivery vehicles. Handb Exp Pharmacol: 263-283, 2007.

28. Ho YC, Chung YC, Hwang SM, Wang KC and Hu YC: Transgene expression and differentiation of baculovirus transduced human mesenchymal stem cells. J Gene Med 7: 860-868, 2005.

29. Knoop K, Kolokythas M, Klutz K, et al: Image-guided, tumor stroma-targeted ${ }^{131} \mathrm{I}$ therapy of hepatocellular cancer after systemic mesenchymal stem cell-mediated NIS gene delivery. Mol Ther 19: 1704-1713, 2011. 